\title{
Neuropeptide Y regulates cholesterol metabolism through Y2 receptor in cell- and SNP-dependent manner
}

\author{
Hidesuke Kaji ${ }^{\text {* }}$ \\ ${ }^{1}$ Division of Physiology and Metabolism, University of Hyogo, Japan
}

Neuropeptide Y (NPY) is well known to stimulate appetite through hypothalamic receptor Y1 [1], while NPY has been reported by Kuo et al. to cause metabolic syndrome through peripheral receptor Y2 (Y2R) under stress and high calorie diet [2]. We have reported the association between $Y 2 R$ promoter SNPs and plasma HDL-cholesterol(HDL-C) levels in healthy human [3]. Y2R gene promoter SNPs rs6857530 G plus rs6857715 T: SNPs $(\mathrm{G}+\mathrm{T})$ was associated with lower levels of plasma HDL-C. SNPs rs6857530 A plus rs6857715 C: SNPs $(A+C)$ was associated with higher levels of plasma HDL-C. These $Y 2 R$ gene promoter SNPs were considered to be a predictor for plasma HDL-C levels independent of sex and plasma triglyceride levels by using multiple regression analysis.

To elucidate the underlying mechanism, activity of gene promoter with each SNPs was assessed by luciferase assay. Luciferase activity using gene promoter containing SNPs $(\mathrm{G}+\mathrm{T})$ was detectable in HepG2, whereas those containing SNPs $(\mathrm{A}+\mathrm{C})$ was detectable in macrophage differentiated from THP-1 cells [4]. Luciferase activity using gene promoter containing neither SNPs $(\mathrm{G}+\mathrm{T})$ nor $(\mathrm{A}+\mathrm{C})$ was detectable in adipocytes differentiated from 3T3-L1 and human vascular endothelial cells HUEhT-1. Mobility shift assay demonstrated complex of HepG2 but not THP-1 nuclear extracts with oligonucleotide containing rs6857530A and the same complex of nuclear extracts of HepG2 or THP-1 with rs6857530G (band a). Mobility shift assay also demonstrated the other complex of HepG2 but not THP-1 nuclear extracts with rs6857530G (band b). If band a includes a repressor and band $b$ includes an activator that is more potent than repressor, it is consistent with SNPs- and cell type-dependent transcriptional activity. Further study is required to clarify transcription factors relevant for determining SNPs-and cell-dependent $Y 2 R$ gene transcriptional activity.

Both HepG2 and macrophage/THP-1 cells contained heterozygous SNPs rs6857715 G/T and rs6857715 A/C. Thus, the effect of potent Y2R antagonist BIIE0246 on transcriptome profiling in these cells was assessed by using microarray. HDL metabolic pathway and sterol responsive element binding protein signaling included genes up- and down-regulated by BIIE0246 in NPY-treated HepG 2 cells, respectively [5]. On the other hand, leptin signaling pathway included genes upregulated by BIIE0246 in NPY-treated macrophage/THP-1 cells [6]. Leptin has been reported to inhibit the activity of acyl coenzyme A:cholesterol acyltransferase 1, thereby inhibiting HDL-mediated cholesterol efflux from macrophages [7].

One target for anti-atherosclerosis is dyslipidemia. To reduce plasma LDL-C levels for prevention of atherosclerotic cardiovascular disease (ASCVD), conventional drugs such as statin and ezetimibe, and new drugs such as proprotein convertase subtilisin/kexin type9(PCSK9) inhibitors to suppress hepatic LDL receptor degradation and microsomal triglyceride transfer protein (MTP) inhibitors to suppress apoB-100 production are being successfully used [8]. Regarding HDL-C, recently developed CETP inhibitors including torcetrapib, dalcetrapib, anacetrapib and evacetrapib caused sufficient elevation of plasma HDL-C levels but did not show any clinical benefit for ASCVD morbidity and mortality [9]. Since HDL has varying functions such as cholesterol efflux, anti-inflammation, anti-oxidation etc. [10], raising the specific HDL quality instead of plasma HDL-C quantity is nowadays being considered more important for ASCVD prevention.Taken together, cell specific modulation of Y2R might be a prospective target for SNP-dependent amelioration of HDL quality with personalized prevention of atherosclerosis.

\section{Acknowledgments}

This study was funded by JSPS KAKENHI Grant Number JP 25504009

\section{References}

1. Kaji H (2013) Neuropeptide $Y$ and its receptors: molecular structure and pathophysiological role in food intake and energy homeostasis. In Neuropeptide $\mathrm{Y}$, molecular structure, role in food intake and direct/indirect effects ed.by Parker SL. Nova Biomedical, New York 39-82.

2. Kuo LE, Kitlinska JB, Tilan JU, Li L, Baker SB, et al. (2007) Neuropeptide Y acts directly in the periphery on fat tissue and mediates stress-induced obesity and metabolic syndrome. Nat Med 13: 803-811. [Crossref]

3. Takiguchi E, Fukano C, Kimura Y, Tanaka M, Tanida K, et al. (2010) Variation in the 5 '-flanking region of the neuropeptide Y2 receptor gene and metabolic parameters. Metabolism 59: 1591-1596. [Crossref]

4. Okada M, Nagai M, Hamaue A, Mori M, Kaji H (2015) Single nucleotide polymorphism- and cell type-dependent gene expression of neuropeptide Y2 receptor. Integr Mol Med 2: 251-255

5. Kaji H, Okada M, Hamaue A, Mori M, Nagai M (2016) Blockade of the neuropeptide Y Y2 receptor with the potent antagonist BIIE0246 regulates gene expression levels in the lipid metabolic pathways in human hepatoma cell line HepG2. Integr Mol Med 3: 576-582.

6. Kaji H, Okada M, Mori M, Hamaue A, Nagai M (2016) Neuropeptide Y regulates leptin signaling pathway via receptor $\mathrm{Y} 2$ assessed by microarray in human macrophage.Int $J$ Biochem Res Rev 15: 1-9.

7. Hongo S, Watanabe T, Arita S, Kanome T, Kageyama H, et al. (2008) Leptin modulates ACAT1 expression and cholesterol efflux from human macrophages. Am J Physiol Endocrinol Metab 297: E474-E482. [Crossref]

8. Chang Y, Robidoux J (2017) Dyslipidemia management update. Curr Opin Pharmacol 33: 47-55. [Crossref]

Correspondence to: Kaji H. M.D., Ph.D.Division of Physiology and Metabolism University of Hyogo, Japan, Tel: Tel: 81-78-925-9421;E-mail: hidesuke_kaji@ cnas.u-hyogo.ac.jp

Received: April 12, 2017; Accepted: April 28, 2017; Published: April 30, 2017 
9. Nicholls SJ, Ray KK, Ballantyne CM, Beacham LA, Miller DL, et al. (2017) Comparative effects of cholesteryl ester transfer protein inhibition, statin or ezetimibe on lipid factors: The ACCENTUATE trial. Atherosclerosis 261: 12-18. [Crossref]
10. Kaji H (2013) High-density lipoproteins and the immune system. J Lipids 2013: 1-8. [Crossref]

Copyright: (2017 Kaji H. This is an open-access article distributed under the terms of the Creative Commons Attribution License, which permits unrestricted use, distribution, and reproduction in any medium, provided the original author and source are credited. 